Tropical Journal of Pharmaceutical Research November 2017; 16 (11): 2735-2740

ISSN: $1596-5996$ (print); 1596-9827 (electronic)

(C) Pharmacotherapy Group, Faculty of Pharmacy, University of Benin, Benin City, 300001 Nigeria.

All rights reserved.

Available online at http://www.tjpr.org

Original Research Article

http://dx.doi.org/10.4314/tjpr.v16i11.22

\title{
Comparison of the effectiveness of polymer gel dosimeters (Magic and Pagatug) for organ dose calculation in brachytherapy, nuclear medicine and teletherapy
}

\author{
Karim Adinehvand ${ }^{1}$, Dariush Sardari ${ }^{1 \star}$, Mohammad Hosntalab ${ }^{1}$ and Majid \\ Pouladian $^{2}$ \\ ${ }^{1}$ Department of Medical Radiation Engineering, ${ }^{2}$ Biomedical Engineering Department, Science and Research Branch, Islamic \\ Azad University, Tehran, Iran \\ *For correspondence: Email: sardari@srbiau.ac.ir
}

\begin{abstract}
Purpose: To investigate and compare two polymer gel dosimeters, Magic and Pagatug, as organ dosimeters for 3D measurement of dose distribution in brachytherapy, nuclear medicine and teletherapy.

Methods: Magic and Pagatug polymer gels were compared with soft tissue based on irradiation with low energy photons during therapeutic applications. Comparison was simulated using Monte-Carlobased MCNPX code. ORNL phantom-Female was used to model some vital organs (kidneys, ovaries and uterus). The right kidney was proposed to be the source of irradiation and the two organs were exposed to this irradiation.

Results: The effective atomic numbers of soft tissue, Magic and Pagatug were 6.86134, 7.07 and 7.2884, respectively. The results showed that Magic and Pagatug, were comparable to soft tissue with regard to application in nuclear medicine and teletherapy. Differences between gel dosimeters and soft tissue were defined as the dose responses. This difference was $<8.1,<4$ and $<76.8 \%$ for teletherapy, nuclear medicine and brachytherapy, respectively.

Conclusion: Due to slight differences between the effective atomic numbers of these polymer gel dosimeters and soft tissue, the polymer gels are not suitable for brachytherapy since the photoelectric interaction is dominant for low energy photons, and the interaction relates to Z4. The results demonstrate that the gel dosimeters are best suited for nuclear medicine.
\end{abstract}

Keywords: Magic, Pagatug, Brachytherapy, Nuclear medicine, Teletherapy, Organ dosimetry, Soft tissue

Tropical Journal of Pharmaceutical Research is indexed by Science Citation Index (SciSearch), Scopus, International Pharmaceutical Abstract, Chemical Abstracts, Embase, Index Copernicus, EBSCO, African Index Medicus, JournalSeek, Journal Citation Reports/Science Edition, Directory of Open Access Journals (DOAJ), African Journal Online, Bioline International, Open-J-Gate and Pharmacy Abstracts

\section{INTRODUCTION}

There are several dosimetry techniques used to determine the distribution of radiation dose during radiation treatment. Each dosimeter tool has a set of unique advantages and disadvantages. Gel dosimeters are appropriate dosimeters for determining 3D dose distribution with high spatial resolution unlike conventional dosimeters such as radioactive ionization chambers, TLD cards and radiography films $[1,2]$.

The TLD cards (also called mini ionization chambers) have some drawbacks in measuring very high doses with high dose gradients, due to their finite sizes which permit measurement of the dose only at a single point [1]. Film batches 
can also offer 3D dose measurements by positioning film in multiple planes but accurate positioning of films in several layers can be a difficult and time-consuming process. Therefore, conventional dosimeters are suitable for clinical brachytherapy [3]. Fricke gel and polymer gel dosimeters are two different dosimeters which can give 3D dose distribution. However, polymer gel dosimeters maintain a high spatial integrity when compared with the Fricke gel dosimeters [4]. There are several different scans in nuclear medicine applications, of which heart scans are the most common. In these scans, the kidneys absorb the highest amount of dose as well as some other critical organs such as ovaries and uterus [5]. For this reason, the source positions are placed in the kidneys in brachytherapy, and the kidneys are radiated in nuclear medicine and teletherapy.

As stated earlier, polymer gels can be used in medical phantoms as valuable tools for determining 3D dose distribution in a medium. Polymer gel dosimeter can replace medical phantom materials as media with features similar to soft tissue. This type of dosimeter can be considered as a suitable option for studying dose distribution in sensitive organs during radiation therapy and nuclear medicine. The dose received in sensitive organs has important role during radiation therapy and nuclear medicine.

Therefore, in the present study, the capabilities of polymer gel dosimeters, Magic and Pagatug as medical phantoms for evaluating absorbed dose of radiation in the ovaries and uterus from the right kidney during brachytherapy, nuclear medicine and teletherapy were investigated.

\section{EXPERIMENTAL}

ORNL phantom-Female was chosen for this study. The phantom consisted of 3 materials and $40+$ discrete cells that simulate the human body [6]. The radiation sources considered for brachytherapy, nuclear medicine and teletherapy are TC-99m [4], Co-60 [7] and ${ }^{125} \mathrm{I}$ [6], respectively.

Table 1 shows the composition of the Magic and Pagatug polymer gel dosimeters, while Table 2 shows the elemental composition of ORNL phantom-Female.

Table 1: Construction of polymer gel dosimeters, Magic and Pagatug

\begin{tabular}{|c|c|c|c|c|c|c|c|c|c|}
\hline Material & $w_{C}$ & $w_{H}$ & $w_{N}$ & $w_{0}$ & $w_{\mathbf{S}}$ & $w_{C u(i i)}$ & $p($ g.cm-3) & $\mathrm{Pe}_{\mathrm{g}}$ & $Z_{\text {eff }}$ \\
\hline Magic[8] & 0.0751 & 0.1062 & 0.0139 & 0.8021 & $2.58 \times 10^{-6}$ & $5.08 \times 10^{-6}$ & 1.06 & 3.51 & 7.07 \\
\hline Pagatug[9] & 0.092 & 0.102 & 0.034 & 0.771 & - & - & 1.0653 & 3.53 & 7.2884 \\
\hline
\end{tabular}

Table 2: Elemental composition of the tissues for ORNL phantom-Female [11]

\begin{tabular}{l|c|c|c}
\hline \multicolumn{4}{c}{ Weight (\%) } \\
\hline Element & Soft tissue & Skeleton & Lung \\
\hline $\mathrm{H}$ & 10.454 & 7.337 & 10.134 \\
$\mathrm{C}$ & 22.663 & 25.475 & 10.238 \\
$\mathrm{~N}$ & 2.490 & 3.057 & 2.866 \\
$\mathrm{O}$ & 63.525 & 47.893 & 75.752 \\
$\mathrm{~F}$ & 0 & 0.025 & 0 \\
$\mathrm{Na}$ & 0.112 & 0.326 & 0.184 \\
$\mathrm{Mg}$ & 0.013 & 0.112 & 0.007 \\
$\mathrm{Si}$ & 0.030 & 0.002 & 0.006 \\
$\mathrm{P}$ & 0.134 & 5.095 & 0.080 \\
$\mathrm{~S}$ & 0.204 & 0.173 & 0.225 \\
$\mathrm{Cl}$ & 0.133 & 0.143 & 0.266 \\
$\mathrm{~K}$ & 0.208 & 0.153 & 0.194 \\
$\mathrm{Ca}$ & 0.024 & 10.190 & 0.009 \\
$\mathrm{Fe}$ & 0.005 & 0.008 & 0.037 \\
$\mathrm{Zn}$ & 0.003 & 0.005 & 0.001 \\
$\mathrm{Rb}$ & 0.001 & 0.002 & 0.001 \\
$\mathrm{Sr}$ & 0 & 0.003 & 0 \\
$\mathrm{Zr}$ & 0.001 & 0 & 0 \\
$\mathrm{~Pb}$ & 0 & 0.001 & 0 \\
$\mathrm{Density}\left(\mathrm{g} / \mathrm{cm}^{3}\right)$ & 1.04 & 1.4 & 0.296 \\
$\mathrm{Z}$ eff & 6.86134 & - & - \\
\hline
\end{tabular}

\section{Simulation procedure}

In this study, MCNPX 2.6.0 was used for the simulations. F4 tallied with DFn, and DEn was used for dose measurement. Figure 1 shows simulated phantom (ORNL phantom-Female) in MCNPX code.

\section{RESULTS}

Outputs of simulations were determined for polymer gel dosimeters and soft tissue during brachytherapy, nuclear medicine and teletherapy, and the results are shown in Table 3 and Table 4.

Table 3 shows that the polymer gel dosimeters, Magic and Pagatug, are comparable to soft tissue for use in nuclear medicine and teletherapy. Differences between gel dosimeters and soft tissue were defined as the dose responses. This difference is $<8.1,<4$ and $<$ $76.8 \%$ for teletherapy, nuclear medicine and brachytherapy, respectively. 

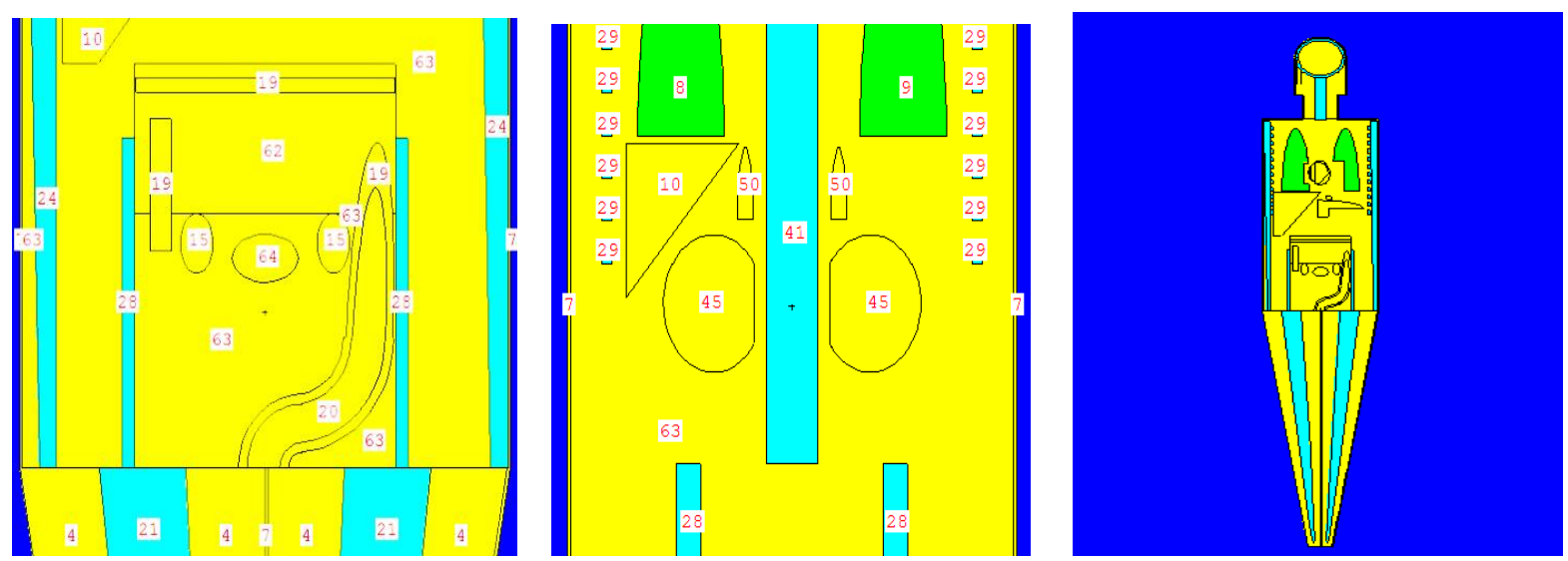

Figure 1: Simulated phantom ORNL phantom-Female (15 Ovaries, 45 Kidneys and 64 Uterus)

Table 3: Output of simulations for polymer gel dosimeters during Brachytherapy, Nuclear medicine and Teletherapy

\begin{tabular}{|c|c|c|c|c|c|c|c|c|}
\hline $\begin{array}{l}\text { Type of } \\
\text { source }\end{array}$ & $\begin{array}{l}\text { Activity } \\
\text { MBq }\end{array}$ & Source position & $\begin{array}{l}\text { Evaluated } \\
\text { organ }\end{array}$ & $\begin{array}{l}\text { Dose in } \\
\text { evaluated } \\
\text { organ } \\
\text { rem/h }\end{array}$ & Error & $\begin{array}{l}\text { Type of } \\
\text { dosimeter }\end{array}$ & $\begin{array}{l}\text { Density } \\
\text { of } \\
\text { dosimeter } \\
g^{r} / \mathrm{cm}^{3}\end{array}$ & $\begin{array}{l}\text { Difference } \\
\text { with soft } \\
\text { tissue \% }\end{array}$ \\
\hline $725 I$ & 1110 & Right kidney & Ovaries & $2.18 \mathrm{E}-2$ & 0.0260 & Magic & 1.095 & 68.5 \\
\hline${ }^{125}$ & 1110 & Right kidney & Uterus & 1.72E-2 & 0.0164 & Magic & 1.095 & 73.3 \\
\hline TC-99m & 1110 & Right kidney & Ovaries & $7.13 \mathrm{E}-2$ & 0.0111 & Magic & 1.095 & 3.4 \\
\hline TC-99m & 1110 & Right kidney & Uterus & $6.29 \mathrm{E}-2$ & 0.0071 & Magic & 1.095 & 3.1 \\
\hline Co-60 & $188 \mathrm{E}+6$ & $\begin{array}{l}100 \mathrm{~cm} \text { from } \\
\text { right kidney }\end{array}$ & Ovaries & $6.94 \mathrm{E}-7$ & 0.0587 & Magic & 1.095 & -8.1 \\
\hline Co-60 & $188 \mathrm{E}+6$ & $\begin{array}{l}100 \mathrm{~cm} \text { from } \\
\text { right kidney }\end{array}$ & Uterus & $6.89 \mathrm{E}-7$ & 0.0404 & Magic & 1.095 & -1.4 \\
\hline${ }^{125}$ I & 1110 & Right kidney & Ovaries & $6.68 \mathrm{E}-3$ & 0.0388 & Pagatug & 1.0653 & 67 \\
\hline${ }^{125} \mathbf{j}$ & 1110 & Right kidney & Uterus & $5.57 \mathrm{E}-3$ & 0.0227 & Pagatug & 1.0653 & 76.8 \\
\hline TC-99m & 1110 & Right kidney & Ovaries & 6.06E-2 & 0.0102 & Pagatug & 1.0653 & 3.9 \\
\hline TC-99m & 1110 & Right kidney & Uterus & 5.30E-2 & 0.0061 & Pagatug & 1.0653 & 3.5 \\
\hline Co-60 & $188 \mathrm{E}+6$ & Right kidney & Ovaries & $6.33 \mathrm{E}-7$ & 0.0569 & Pagatug & 1.0653 & -8.1 \\
\hline Co-60 & $188 \mathrm{E}+6$ & Right kidney & Uterus & $6.30 \mathrm{E}-7$ & 0.0405 & Pagatug & 1.0653 & -1.5 \\
\hline
\end{tabular}

Table 4: Output of simulations for soft tissue during brachytherapy, nuclear medicine and teletherapy

\begin{tabular}{|c|c|c|c|c|c|}
\hline $\begin{array}{l}\text { Type of } \\
\text { source }\end{array}$ & $\begin{array}{l}\text { activity } \\
\mathrm{MBq}\end{array}$ & Source position & $\begin{array}{l}\text { Evaluated } \\
\text { organ }\end{array}$ & $\begin{array}{l}\text { Dose in evaluated } \\
\text { organ } \\
\text { rem/h }\end{array}$ & Error \\
\hline Co-60 & $188 \mathrm{E}+6$ & $100 \mathrm{~cm}$ away from right kidney & Ovaries & $6.89 \mathrm{E}-7$ & 0.0557 \\
\hline Co-60 & $188 \mathrm{E}+6$ & $100 \mathrm{~cm}$ away from right kidney & Uterus & $6.40 \mathrm{E}-7$ & 0.0401 \\
\hline${ }^{125} \mid$ & 1110 & Right kidney & Ovaries & $4.00 \mathrm{E}-3$ & 0.0228 \\
\hline${ }^{125} \mathbf{I}$ & 1110 & Right kidney & Uterus & $3.15 \mathrm{E}-3$ & 0.0127 \\
\hline TC-99m & 1110 & Right kidney & Ovaries & $5.83 \mathrm{E}-2$ & 0.0046 \\
\hline TC-99m & 1110 & Right kidney & Uterus & $5.12 \mathrm{E}-2$ & 0.0027 \\
\hline
\end{tabular}

Table 5: Comparison between errors (\%) of Magic and Pagatug Gel dosimeters

\begin{tabular}{lcc}
\hline Type of treatment & $\begin{array}{c}\text { Max. error } \\
\text { in } \\
\text { using } \\
\text { MAGIC }\end{array}$ & $\begin{array}{c}\text { Max. error in } \\
\text { using } \\
\text { PAGATUG }\end{array}$ \\
\hline Teletherapy & 8.1 & 8.1 \\
Nuclear medicine & 3.4 & 3.9 \\
Brachytherapy & 68.5 & 76.8 \\
\hline
\end{tabular}

The results demonstrate that the gel dosimeters are best suited for nuclear medicine.

Table 6, Table 7 and Table 8 show the ratios of photon interactions for the polymer gel dosimeters and soft tissue during brachytherapy, nuclear medicine and teletherapy. These results show that the dominant interaction in the polymer gel dosimeters and soft tissue for nuclear medicine and teletherapy was Compton 
Table 6: Photon with polymer gel dosimeter and soft tissue interactions ratio for Brachytherapy

\begin{tabular}{|c|c|c|c|c|c|}
\hline \multicolumn{6}{|l|}{ Brachytherapy } \\
\hline \multicolumn{6}{|l|}{ Pagatug } \\
\hline Flux(photon) & $1.11 \mathrm{E}-5$ & Ovaries & Flux (photon) & $9.23 \mathrm{E}-6$ & Uterus \\
\hline Reaction rate & $2.15 \mathrm{E}-6$ & Incoherent & Reaction rate & $1.79 \mathrm{E}-6$ & Incoherent \\
\hline Reaction rate & 4.87E-7 & Coherent & Reaction rate & $4.05 \mathrm{E}-7$ & Coherent \\
\hline Reaction rate & $1.36 \mathrm{E}-6$ & Photoelectric & Reaction rate & $1.14 \mathrm{E}-6$ & Photoelectric \\
\hline Reaction rate & 0.00 & Pair Production & Reaction rate & 0.00 & Pair Production \\
\hline Reaction rate & $4.00 \mathrm{E}-6$ & Total & Reaction rate & 3.33E-6 & Total \\
\hline \multicolumn{6}{|l|}{ Magic } \\
\hline Flux (photon) & $1.11 \mathrm{E}-5$ & Ovaries & Flux(photon) & $9.09 \mathrm{E}-6$ & Uterus \\
\hline Reaction rate & $2.15 \mathrm{E}-6$ & Incoherent & Reaction rate & $1.76 \mathrm{E}-6$ & Incoherent \\
\hline Reaction rate & $4.92 E-7$ & Coherent & Reaction rate & $3.98 \mathrm{E}-7$ & Coherent \\
\hline Reaction rate & $1.40 \mathrm{E}-6$ & Photoelectric & Reaction rate & $1.13 \mathrm{E}-6$ & Photoelectric \\
\hline Reaction rate & 0.00 & Pair Production & Reaction rate & 0.00 & Pair Production \\
\hline \multirow[t]{2}{*}{ Reaction rate } & $4.05 \mathrm{E}-6$ & Total & Reaction rate & $3.28 \mathrm{E}-6$ & Total \\
\hline & & Soft Tissue & & & \\
\hline Flux (photon) & $6.84 \mathrm{E}-6$ & Ovaries & Flux(photon) & $5.45 \mathrm{E}-6$ & Uterus \\
\hline Reaction rate & $1.16 \mathrm{E}-6$ & Incoherent & Reaction rate & $9.28 \mathrm{E}-7$ & Incoherent \\
\hline Reaction rate & $3.15 \mathrm{E}-7$ & Coherent & Reaction rate & $2.48 \mathrm{E}-7$ & Coherent \\
\hline Reaction rate & $1.11 \mathrm{E}-6$ & Photoelectric & Reaction rate & $8.51 \mathrm{E}-7$ & Photoelectric \\
\hline Reaction rate & 0.00 & Pair Production & Reaction rate & 0.00 & Pair Production \\
\hline Reaction rate & $2.59 \mathrm{E}-6$ & Total & Reaction rate & $2.03 \mathrm{E}-6$ & Total \\
\hline
\end{tabular}

Table 7: Photon with polymer gel dosimeter and soft tissue interactions ratio for nuclear medicine

\begin{tabular}{|c|c|c|c|c|c|}
\hline \multicolumn{6}{|c|}{ Nuclear medicine } \\
\hline \multicolumn{6}{|l|}{ Pagatug } \\
\hline Flux(photon) & $1.78 \mathrm{E}-4$ & Ovaries & Flux (photon) & $1.55 \mathrm{E}-4$ & Uterus \\
\hline Reaction rate & $3.19 \mathrm{E}-5$ & Incoherent & Reaction rate & $2.78 E-5$ & Incoherent \\
\hline Reaction rate & $2.19 \mathrm{E}-6$ & Coherent & Reaction rate & $1.97 \mathrm{E}-6$ & Coherent \\
\hline Reaction rate & $2.96 \mathrm{E}-6$ & Photoelectric & Reaction rate & $2.70 \mathrm{E}-6$ & Photoelectric \\
\hline Reaction rate & 0.00 & Pair Production & Reaction rate & 0.00 & Pair Production \\
\hline Reaction rate & $3.71 \mathrm{E}-5$ & Total & Reaction rate & $3.25 \mathrm{E}-5$ & Total \\
\hline \multicolumn{6}{|l|}{ Magic } \\
\hline Flux (photon) & $1.77 \mathrm{E}-4$ & Ovaries & Flux (photon) & $1.55 \mathrm{E}-4$ & Uterus \\
\hline Reaction rate & $3.18 \mathrm{E}-5$ & Incoherent & Reaction rate & $2.77 \mathrm{E}-5$ & Incoherent \\
\hline Reaction rate & $2.18 \mathrm{E}-6$ & Coherent & Reaction rate & $1.96 \mathrm{E}-6$ & Coherent \\
\hline Reaction rate & $2.94 \mathrm{E}-6$ & Photoelectric & Reaction rate & 2.67E-6 & Photoelectric \\
\hline Reaction rate & 0.00 & Pair Production & Reaction rate & 0.00 & Pair Production \\
\hline \multirow[t]{2}{*}{ Reaction rate } & $3.69 \mathrm{E}-5$ & Total & Reaction rate & $3.24 \mathrm{E}-5$ & Total \\
\hline & & \multicolumn{2}{|l|}{ Soft Tissue } & & \\
\hline Flux (photon) & $1.74 \mathrm{E}-4$ & Ovaries & Flux (photon) & $1.53 \mathrm{E}-4$ & Uterus \\
\hline Reaction rate & $2.74 \mathrm{E}-5$ & Incoherent & Reaction rate & $2.41 \mathrm{E}-5$ & Incoherent \\
\hline Reaction rate & $2.02 \mathrm{E}-6$ & Coherent & Reaction rate & $1.81 \mathrm{E}-6$ & Coherent \\
\hline Reaction rate & $3.12 E-6$ & Photoelectric & Reaction rate & 2.82E-6 & Photoelectric \\
\hline Reaction rate & 0.00 & Pair Production & Reaction rate & 0.00 & Pair Production \\
\hline Reaction rate & $3.26 \mathrm{E}-5$ & Total & Reaction rate & 2.87E-5 & Total \\
\hline
\end{tabular}

(incoherent) scattering, while in brachytherapy, both Compton (incoherent) and photoelectric were the dominant interactions. The similarity in dominant interactions for the polymer gels and soft tissue shows a good agreement which is of interest for the dosimetry applications.

\section{DISCUSSION}

In this study, the capabilities of Magic and Pagatug polymer gel dosimeters for measuring
3D dose distribution during brachytherapy, nuclear medicine and teletherapy were evaluated by MCNPX code, and their dose responses were compared with soft tissue. These polymer gel dosimeters are capable of measuring complex 3D dose distributions with high spatial resolution, unlike other radiation dosimeters such as ionization chambers, TLD and radiographic films. The results showed that the dosimeters were more suitable for use in teletherapy and nuclear medicine when compared as opposed to brachytherapy. In brachytherapy the two gel 
Table 8: Photon with polymer gel dosimeter and soft tissue interactions ratio for teletherapy

\begin{tabular}{|c|c|c|c|c|c|}
\hline \multicolumn{6}{|l|}{ Pagatug } \\
\hline Flux (photon) & $9.08 \mathrm{E}-15$ & Ovaries & Flux(photon) & $9.00 \mathrm{E}-15$ & Uterus \\
\hline Reaction rate & $1.49 E-15$ & Incoherent & Reaction rate & $1.49 \mathrm{E}-15$ & Incoherent \\
\hline Reaction rate & $6.04 \mathrm{E}-17$ & Coherent & Reaction rate & $6.74 \mathrm{E}-17$ & Coherent \\
\hline Reaction rate & $6.18 \mathrm{E}-17$ & Photoelectric & Reaction rate & 7.93E-17 & Photoelectric \\
\hline Reaction rate & 0.00 & Pair Production & Reaction rate & 0.00 & Pair Production \\
\hline Reaction rate & $1.61 \mathrm{E}-15$ & Total & Reaction rate & $1.63 \mathrm{E}-15$ & Total \\
\hline \multicolumn{6}{|l|}{ Magic } \\
\hline Flux (photon) & $9.08 \mathrm{E}-15$ & Ovaries & Flux(photon) & $9.03 E-15$ & Uterus \\
\hline Reaction rate & $1.49 \mathrm{E}-15$ & Incoherent & Reaction rate & $1.49 \mathrm{E}-15$ & Incoherent \\
\hline Reaction rate & $6.10 \mathrm{E}-17$ & Coherent & Reaction rate & $6.77 \mathrm{E}-17$ & Coherent \\
\hline Reaction rate & $6.40 \mathrm{E}-17$ & Photoelectric & Reaction rate & $7.91 \mathrm{E}-17$ & Photoelectric \\
\hline Reaction rate & 0.00 & Pair Production & Reaction rate & 0.00 & Pair Production \\
\hline Reaction rate & $1.61 \mathrm{E}-15$ & Total & Reaction rate & $1.64 \mathrm{E}-15$ & Total \\
\hline & & \multicolumn{2}{|l|}{ Soft tissue } & & \\
\hline Flux (photon) & $9.81 \mathrm{E}-15$ & Ovaries & Flux (photon) & $9.08 \mathrm{E}-15$ & Uterus \\
\hline Reaction rate & $1.42 \mathrm{E}-15$ & Incoherent & Reaction rate & $1.32 E-15$ & Incoherent \\
\hline Reaction rate & $6.68 \mathrm{E}-17$ & Coherent & Reaction rate & $6.41 \mathrm{E}-17$ & Coherent \\
\hline Reaction rate & $8.49 E-17$ & Photoelectric & Reaction rate & $9.18 \mathrm{E}-17$ & Photoelectric \\
\hline Reaction rate & 0.00 & Pair Production & Reaction rate & 0.00 & Pair Production \\
\hline Reaction rate & $1.58 \mathrm{E}-15$ & Total & Reaction rate & $1.48 \mathrm{E}-15$ & Total \\
\hline
\end{tabular}

dosimeters had more than 10 percent error, which is not acceptable in brachytherapy. The difference observed between the gel dosimeters and soft tissue is related to incoherent interaction with $48.1 \%$ deviation. In teletherapy, both Magic and Pagatug had similar errors and they were both reliable. Photoelectric interaction is the main cause of difference between polymer gels and soft tissue in teletherapy. In nuclear medicine, Magic has less error when compared to Pagatug, due to differences between their effective atomic numbers. The effective atomic numbers for soft tissue, Pagatug and Magic were 6.86134, 7.2884 and 7.07 , respectively.

It is known that photoelectric interaction is dominant for lower energy photons and this interaction relates to atomic number as $Z^{4}[10]$. Since the effective atomic numbers of soft tissue and the gel dosimeters were different, larger errors would occur in lower energy photons. Hence, the dose response measurement error for Pagatug polymer gel was larger than that for Magic polymer gel.

\section{CONCLUSION}

The results obtained in this investigation strongly suggest that if low energy sources are required in therapeutic applications, Magic and Pagatug polymer gel dosimeters are acceptable soft tissue equivalent dosimeters.

\section{DECLARATIONS}

\section{Conflict of Interest}

No conflict of interest associated with this work.

\section{Contribution of Authors}

The authors declare that this work was done by the authors named in this article and all liabilities pertaining to claims relating to the content of this article will be borne by them.

\section{Open Access}

This is an Open Access article that uses a funding model which does not charge readers or their institutions for access and distributed under the terms of the Creative Commons Attribution License (http://creativecommons.org/licenses/by/ 4.0) and the Budapest Open Access Initiative (http://www.budapestopenaccessinitiative.org/rea d), which permit unrestricted use, distribution, and reproduction in any medium, provided the original work is properly credited.

\section{REFERENCES}

1. Shani G. Radiation dosimetry instrumentation and methods. CRC Press; 2000 Dec 28.

2. Venning $A$, Healy $B$, Nitschke $K$, Baldock $C$. Investigation of the MAGAS normoxic polymer gel dosimeter with Pyrex glass walls for clinical radiotherapy dosimetry. Nucl Instrum Methods Phys Res A 2005; 555: 396-402.

3. Kalaiselven SKD, Rajan JJSE. Polymer Gel Dosimetry for Radiation Therapy. Modern Practices in Radiation Therapy, Natanasabapathi G, Ed. InTech, 2012.

4. Hurley $C$, Venning $A$, Baldock $C$. A study of a normoxic polymer gel dosimeter comprising methacrylic acid, gelatin and tetrakis (hydroxymethyl) phosphonium chloride (MAGAT). Appl Radiat Isot 2005; 63: 443-456. 
5. Sahebnasagh A, Adinehvand $K$, Azadbakht $B$. Determination and Comparison of Absorbed dose of Ovaries and Uterus in Heart Scan from TC-99 m, by Three Methods: TLD Measurement, MCNP Simulation and MIRD Calculation and Estimation of its Risks. Res J Appl Sci Eng Tech 2012; 4: 4572-4575.

6. Lazarine Alexis D. Medical Physics Calculations with Mcnptm: A Primer. Master of Science, Health Physics, Texas A\&M University; 2006.

7. Baumgartner A, Hranitzky C, Stadtmann H, Maringer FJ. Determination of photon fluence spectra from a $60 \mathrm{Co}$ therapy unit based on PENELOPE and MCNP simulations. Radiat Meas 2011; 46: 595-601.
8. Peter MF, Derek CK, Mark DD, John CG. Polymer gels for magnetic resonance imaging of radiation dose distributions at normal room atmosphere. Phys Med Biol 2001; 46: 3105.

9. Abtahi SM, Aghamiri SMR, Khalafi H. Optical and MRI investigations of an optimized acrylamide-based polymer gel dosimeter. J Radioanal Nucl Chem 2014; 300: 287-301.

10. Health Physics. Herman Cember, Thomas E. Johnson, 4th edn, 2012.

11. http://ordose.ornl.gov/resources/Mird.pdf 